\title{
Hamiltonian Description of Composite Fermions: Calculation of Gaps
}

\author{
Ganpathy Murthy ${ }^{a}$ and R.Shankar ${ }^{b}$ \\ (a) Physics Department, Boston University, Boston MA 02215 \\ and Department of Physics and Astronomy, Johns Hopkins University, Baltimore MD 21218; \\ (b) Department of Physics, Yale University, New Haven CT 06520
}

(August 9, 2018)

\begin{abstract}
We analytically calculate gaps for the $\nu=\frac{1}{3}, \frac{2}{5}$, and the $\frac{3}{7}$ polarized and partially polarized Fractional Quantum Hall (FQH) states based on the Hamiltonian Chern-Simons theory we have developed. For a class of potentials that are soft at high momenta (due to the finite thickness of the sample) we find good agreement with numerical and experimental results.
\end{abstract}

73.50.Jt, 05.30.-d, 74.20.-z

The Composite Fermion(CF) picture [1] has been a very successful organizing principle in understanding the Fractional Quantum Hall effects(FQHE) [2] as well as in generating wavefunctions for these states [3]. ChernSimons(CS) field theories [4 have provided us with a link between the microscopic formulation of the problem and experiment, both for incompressible and compressible states [9, 10].

Recently we developed a hamiltonian CS theory for the FQH states [11, 12]. Inspired by the work of Bohm and Pines 13 on the 3D electron gas, we enlarged the Hilbert space to introduce $n$ high-energy magnetoplasmons degrees of freedom, $(n$ also being the number of electrons) at the same time imposing an equal number of constraints on physical states. Upon ignoring the coupling between the oscillators and the fermions we obtained some well known wavefunctions 14,1]. However the fermions still had the bare mass, and the frequency of the magnetoplasmons was incorrect. Hence a final canonical transformation was employed to decouple the fermions from the oscillators in the infrared limit.

We choose to call the final fermions the composite fermions for the following reasons. First, the final canonical transformation assigns to each fermion the magnetic moment $e / 2 m$ as mandated by the arguments of Refs. 115,16. Next, the fermions have $1 / m^{*}=0$ in the absence of interactions and acquire an interaction dependent $1 / m^{*}$. Finally, the formula for the electronic charge density takes the following form, separable into high- and low-energy pieces 12, at small $q$ :

$$
\begin{array}{r}
\rho_{e}(q)=\frac{q}{\sqrt{8 \pi}} \sqrt{\frac{2 p}{2 p+1}}\left(A(q)+A^{\dagger}(-q)\right) \\
+\frac{\sum_{j} e^{-i q x_{j}}}{2 p+1}-i l^{2}\left(\sum_{j}\left(q \times \Pi_{j}\right) e^{-i q x_{j}}\right)
\end{array}
$$

where $A$ refers to the oscillators, $l=1 / \sqrt{e B}$ is the magnetic length, and $\vec{\Pi}_{j}=\vec{P}_{j}+e \vec{A}^{*}\left(r_{j}\right)$ is the velocity operator of the CFs. The oscillator piece saturates Kohn's theorem [18], and the rest, to be called $\bar{\rho}$, satisfies the magnetic translation algebra [19 to lowest leading order. Note that $\bar{\rho}$ is a sum of a monopole with charge $e^{*}=e /(2 p+1)$, which is the charge associated with the $\mathrm{CF}$, and a dipole piece which alone survives at $\nu=1 / 2$ and has the value proposed by Read [17. (A number of recent constructions have emphasized this dipolar aspect [20.21).

The Hamiltonian of the low-energy sector (dropping the magnetic moment term) is

$$
H=\frac{1}{2} \int \frac{d^{2} q}{(2 \pi)^{2}} v(q) \bar{\rho}(-q) \bar{\rho}(q) .
$$

where $v(q)$ is the electron-electron interaction. This hamiltonian is to be supplemented by $n$ constraints (which will be ignored in the earlier parts of this discussion).

Earlier, we extracted [11.12 from this Hamiltonian a "free-particle" part, (corresponding to the diagonal terms in the sum over particles ) and thereby an effective mass for the CFs, which we found (for a Coulomb interaction cutoff at wave vector $\left.Q=k_{F}=1 / l\right)$ to be $\frac{1}{m^{*}} \simeq \frac{e^{2}}{\epsilon l} \cdot \frac{1}{6}$ where $\epsilon$ is the dielectric constant. However, we emphasized 12 that since the interactions were of the same magnitude as the "free-particle" term and could renormalize this mass considerably, one should calculate directly observable quantities using the full Hamiltonian. This is what we shall do now by computing transport gaps for a few fractions. Since the CF already has many of the nonperturbative charge and mass renormalizations built into it, we can expect that we may pass readily from the hamiltonian to observables using a variety of approximation methods.

We now describe our calculation of gaps for $\nu=$ $\frac{1}{3}, \frac{2}{5}, \frac{3}{7}$ in the Hartree-Fock (HF) approximation. The gaps are given by the difference in the expectation of $H$ between the ground state and a state with a widely separated particle-hole pair. For the states that appear in the calculation, we use the ones that appear naturally in $\mathrm{CF}$ theory [1], which are just the eigenstates of the freeparticle part alluded to earlier: $p$ filled CF- Landau levels (CF-LLs) for the ground state, and states with particlehole excitations above it.

Note that we do not convert these $\mathrm{CF}$ wavefunctions to electronic ones (by multiplying by Jastrow factors and 
projecting to the LLL). Instead we use our formula for the electronic charge operator $\bar{\rho}$ (in which these effects are subsumed by the reduced charge $e^{*}$ and the dropping of the oscillator part) and write $H$ in terms of it. We circumvent the fact that $\bar{\rho}$ is to be trusted only for $q l \ll 1$ as follows. Consider real samples which have a finite thickness $\Lambda$ of the same order as $l$, so that the Coulomb interaction is cutoff at large $q$ [22]. It was realized by Haldane and Rezayi [23] that this has a large effect on the gap, while leaving the wavefunctions essentially unchanged. We will focus on such interactions, parametrized by $\lambda=\Lambda / l$ for which numerical results are available [24 26]. The advantage is that as $\lambda$ becomes large only small- $q$ matrix elements of the density are invoked in computing gaps, and we expect our theory to become more accurate. It is possible that beyond some large $\lambda$ the liquid state might cease to be the ground state. Our theory, which is based on a liquid state with uniform density, can be expected to work up to this $\lambda$.

We work in the symmetric gauge, in which the singleparticle wavefunctions are characterized by the LL index $n$ and the angular momentum index $m$. The formalism in this gauge has been extensively developed 14,27]. The magnetoexciton wave functions [28] $\psi_{n}^{n^{\prime}}\left(q ; \vec{r}, \overrightarrow{r^{\prime}}\right)$ describe a particle in the $n^{\text {th }}$ LL and a hole in the $n^{\prime t h} \mathrm{LL}$, with a conserved momentum $\vec{q}$. In a second quantized notation we can introduce creation $\left(d_{n m}^{\dagger}\right)$ and destruction operators $\left(d_{n m}\right)$, and write the magnetoexciton creation operator as $X_{n}^{n^{\prime}}(q)=\sum_{m m^{\prime}} x_{n n^{\prime}}^{m m^{\prime}}(q) d_{n m}^{\dagger} d_{n^{\prime} m^{\prime}}$ where the $x$-coefficients are (for $m \geq m^{\prime}$ )

$$
\begin{aligned}
x_{n n^{\prime}}^{m m^{\prime}}(q)= & (-1)^{n^{\prime}} \frac{\sqrt{2 \pi}}{L} \sqrt{\frac{m^{\prime} !}{m !}}\left(\frac{i Q_{+}}{\sqrt{2}}\right)^{m-m^{\prime}} \\
& \times L_{m^{\prime}}^{m-m^{\prime}}(y) e^{-y / 2}
\end{aligned}
$$

Here $Q_{+}=l^{*}\left(q_{x}+i q_{y}\right), l^{*}=l \sqrt{2 p+1}$ is the magnetic length in the effective field, $L_{n}^{m}$ is a Laguerre polynomial, and $y=Q^{2} / 2$. The density operator can now be written in terms of the above operators as

$$
\begin{aligned}
& \bar{\rho}(q)=\sum_{n n^{\prime}} \rho_{n}^{n^{\prime}}(q) X_{n}^{n^{\prime}}(q) \\
& \rho_{n}^{n^{\prime}}(q)=\frac{(-1)^{n^{\prime}+1}}{2 p+1} \frac{L}{\sqrt{2 \pi}} \sqrt{\frac{n^{\prime} !}{n !}}\left(\frac{-i Q+}{\sqrt{2}}\right)^{n-n^{\prime}} \\
& \times e^{-y / 2}\left(n L_{n^{\prime}-1}^{n-n^{\prime}}+2 L_{n^{\prime}}^{n-n^{\prime}}-\left(n^{\prime}+1\right) L_{n^{\prime}+1}^{n-n^{\prime}}\right)
\end{aligned}
$$

Apart from the trivial dependence of $x$ on $n^{\prime}$, we see the separation between the angular $m$ labels and the "radial" $n$ labels. We find that that as $\vec{q} \rightarrow 0$ all transition matrix elements vanish at least as $q^{2}$, an essential property of charge density matrix elements in the LLL [19].

We will need the following identities;

$$
\begin{gathered}
\sum_{m} x_{n n}^{m m}(\vec{q})=(-1)^{n} \quad \frac{L}{\sqrt{2 \pi}} \quad \delta_{\vec{Q}, 0} \\
\sum_{m} x_{n_{1} n}^{m_{1} m}\left(\vec{q}_{1}\right) x_{n n_{2}}^{m m_{2}}\left(\vec{q}_{2}\right)=(-1)^{n} \frac{\sqrt{2 \pi}}{L} e^{\frac{-i}{2} \vec{Q}_{1} \times \vec{Q}_{2}} \times \\
x_{n_{1} n_{2}}^{m_{1} m_{2}}\left(\vec{q}_{1}+\vec{q}_{2}\right)
\end{gathered}
$$

The ground state we have assumed is not an eigenstate of $H$, which can create particle-hole pairs above it. However, for rotationally invariant interactions, we find that the Hamiltonian does not mix a single-particle (or singlehole) state with any other single-particle (or single-hole) state, the signature of a HF state. This supports our view that we are working with the right variables.

Consider transport gaps in the spin-polarized states of $\frac{1}{3}, \frac{2}{5}$, and $\frac{3}{7}$. We will work with the model potential $v(r)=e^{2} / \varepsilon \sqrt{r^{2}+\Lambda^{2}}$, whose Fourier transform is $v(q)=2 \pi e^{2} \exp (-q \Lambda) / \varepsilon q$ where $\Lambda$ is the thickness. In order to compute the gap to a neutral excitation (with widely separated quasiparticle and quasihole), which corresponds experimentally to the transport gap, we compute the energy to add a single CF to the $p^{t h}$ LL, and add to it the energy to remove a CF from the $(p-1)^{t h}$ LL. To illustrate how the calculation goes, we work out the case of the quasihole energy for $\nu=\frac{1}{3}$. The quasihole state is $d_{0 m} \mid \Omega>$, where $\mid \Omega>$ denotes the GS. Now one has to compute

$$
\begin{gathered}
\sum_{n_{i} n_{i}^{\prime}, m_{i} m_{i}^{\prime}} x_{n_{1} n_{1}^{\prime}}^{m_{1} m_{1}^{\prime}}(-q) x_{n_{2} n_{2}^{\prime}}^{m_{2} m_{2}^{\prime}}(q) \rho_{n_{1}}^{n_{1}^{\prime}}(-q) \rho_{n_{2}}^{n_{2}^{\prime}}(q) \\
<\Omega\left|d_{0 m}^{\dagger} d_{n_{1} m_{1}}^{\dagger} d_{n_{1}^{\prime} m_{1}^{\prime}} d_{n_{2} m_{2}}^{\dagger} d_{n_{2}^{\prime} m_{2}^{\prime}} d_{m 0}\right| \Omega>_{c}
\end{gathered}
$$

where the subscript $c$ on the expectation value denotes that the operators of the external particle are not allowed to contract with themselves (this corresponds to subtracting the GS energy). Now one performs the standard Wick contractions, with the proviso that two operators belonging to the same density are not allowed to contract with each other (this would correspond to the $q=0$ density, which is cancelled by the background). After performing the Wick contractions one is left with sums over the angular momentum indices, which can be performed by using Eqs.(5).

Similar considerations hold for the quasiparticle energy. Finally, adding the two together to obtain the gap, leads to the following expressions

$$
\begin{array}{cc}
\Delta\left(\frac{1}{3}\right) & =\quad K_{3}\left(\frac{l^{* 3}}{\Lambda^{3}}+h_{0}+2 h_{1}+h_{2}-h_{3}\right) \\
\Delta\left(\frac{2}{5}\right) & =K_{5}\left(\frac{l^{* 3}}{\Lambda^{3}}+h_{0}+4 h_{1}+\frac{3}{2} h_{2}-10 h_{3}+\frac{9}{2} h_{4}-\frac{1}{2} h_{5}\right) \\
\Delta\left(\frac{3}{7}\right) & =K_{7}\left(\frac{l^{* 3}}{\Lambda^{3}}+h_{0}+6 h_{1}+h_{2}-\frac{104}{3} h_{3}+\frac{425}{12} h_{4}-\frac{25}{2} h_{5}\right. \\
+ & \left.\frac{7}{4} h_{6}-\frac{1}{12} h_{7}\right) \\
h_{n}=\frac{1}{K_{p}(2 p+1)^{2}} \int \frac{d^{2} q}{(2 \pi)^{2}} v(q) y^{n} e^{-y}
\end{array}
$$

where $K_{p}=e^{2} /\left(\varepsilon l(2 p+1)^{5 / 2}\right)$, and $y=q^{2} l^{* 2} / 2$. We can compute the gaps for any potential that has its third moment finite [29] (this leads to the first term in the gaps).

Recently, numerical calculations based on $\mathrm{CF}$ wavefunctions have been performed [25,26] on the model potential. Since these wave functions have essentially perfect overlap with exact ground states [3], we surmise that the results are essentially exact. Bearing in mind that our 
results are to be trusted for large $\lambda=\Lambda / l$, we find for all three fractions an error of about $60 \%$ at $\lambda=2$ which decreases to about $30 \%$ for $\lambda=3$.

Table I compares our numbers to those measured on an experimental sample 30], whose $\lambda$ has been determined as explained in ref. [26], based on the detailed calculations of ref. 31]. The difference $(\simeq 10-30 \%)$ between Ref. 26] and experiment is presumably due to disorder, Landaulevel mixing etc.

\begin{tabular}{|c|c|c|c|c|c|}
\hline$\nu$ & $\lambda$ & Ref. [30] & Ref. [26] & Ours1 & Ours2 \\
\hline $1 / 3$ & 2.5 & .042 & .046 & .066 & .053 \\
\hline $2 / 5$ & 2.3 & .017 & .021 & .031 & .020 \\
\hline $3 / 7$ & 2.2 & .010 & .015 & .020 & .011 \\
\hline
\end{tabular}

Table I: Gaps in units of $e^{2} / \varepsilon l$. The comparison is between experiment (Ref. [30]), numerical work (Ref. [26]), and the results of the preceding calculations. The last column corresponds to our results with constraints, to be explained later.

We have also computed the gap to the spin-reversed quasiparticle for $\nu=\frac{1}{3}$ (ignoring the Zeeman energy), $\Delta_{S R}=K_{3}\left(h_{0}+2 h_{1}+h_{2}\right)$. The only results we are aware of for the model potential(ref( 25]) give values for this quantity for $\lambda=0,1.5$. The error at 1.5 is about $30 \%$.

Apart from numbers for the gaps, we have also derived scaling relations between gaps for $p /(2 p+1)$ and $p /(2 s p+$ $1)$, which we will present in detail elsewhere [32].

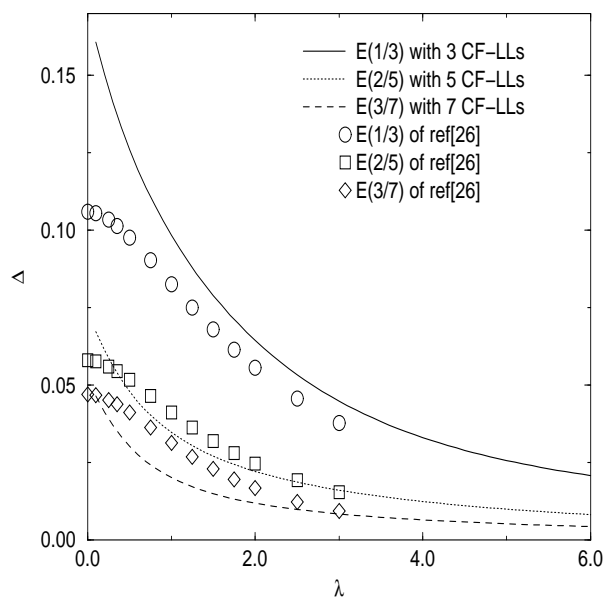

FIG. 1. Gaps to polarized $\frac{1}{3}, \frac{2}{5}$, and $\frac{3}{7}$ states and comparison to results of ref.[26]. All energies are in units of $e^{2} / \varepsilon l$.

Now let us consider the constraints. The divergence of gaps at small $\lambda$ comes from summing over all CFLLs in the sum over intermediate states. We know that the Hilbert space of free fermions is too large for this problem, which is restricted to the electronic LLL. It is the role of the constraints, ignored so far, to select the suitable subspace. In an ideal calculation, a gauge invariant charge operator acting on a physical gauge invariant state will not mix it with intermediate states which are unphysical. There will be no need to invoke constraints. Given that our $\bar{\rho}$ obeys the magnetic algebra when the commutators are calculated in the big space with no regard to constraints, we may conclude it is gauge invariant. However, there are two problems: It represents the density only for small $q$, and the trial states we sandwich it between are free particle, non-gauge invariant, states.

While we do not know which states will be selected by the constraints, we know that (i) in numerical work 33. the low-lying states are in one-to-one correspondence with those of independent CFs, and (ii) the number of constraints is so as to limit the single particle states to those of one filled electronic LL or $2 p+1$ filled CF levels. This suggests a simple, approximate way of dealing with the constraints: keep the first $2 p+1$ lowest-lying CF-LL states. Our results, as shown in Fig.1 in this approximation, are in reasonable agreement with those of ref. 26] over a whole range of $\lambda$. The last column of Table I displays the numbers for the appropriate $\lambda$. While the agreement is gratifying, this is not a controlled procedure, and we cannot be sure that other quantities computed this way will come out with the same accuracy.

We have also computed gaps to singlet (for $\frac{2}{5}$ ) and partially polarized states (for $\frac{3}{7}$ ), ignoring the Zeeman energy, and imposing the constraint as described above. We choose the many-body GS for the singlet $\frac{2}{5}$ state in accordance with CF theory [1] to be the $n=0$ CF-LL occupied with both $\uparrow$ and $\downarrow$ spin CFs. There is only a single energy gap in this system, since both the particle and hole energies are symmetric in spin. For partially polarized $\frac{3}{7}$ we choose the GS to be the $n=0$ CF-LL occupied by both spins, while the $n=1$ CF-LL is occupied by $\uparrow$ spins 11 . In the latter case there are distinct energies for the four possible excitations, with the particle being $\uparrow(n=2$ CF-LL $)$, or $\downarrow$ ( $n=1$ CF-LL $)$, and the hole being $\uparrow(n=1$ CF-LL $)$ or $\downarrow$ ( $n=0$ CF-LL $)$. Our results are presented in Fig.2. We are not aware of calculations of these quantities for the model potential.

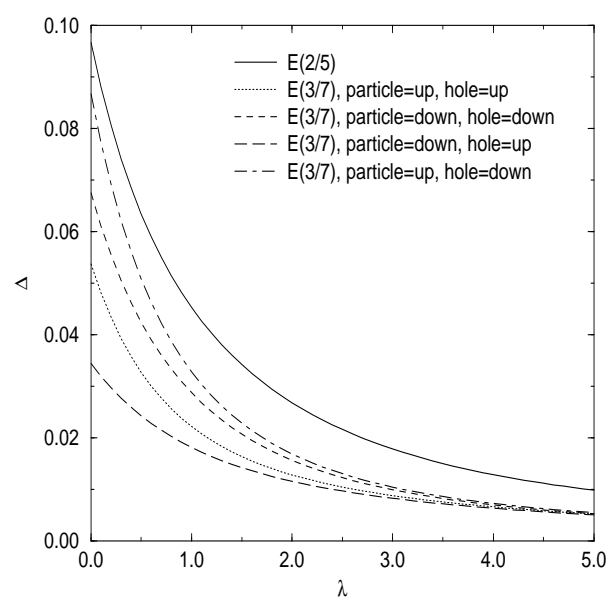

FIG. 2. Gaps to singlet $\left(\frac{2}{5}\right)$ and partially polarized $\left(\frac{3}{7}\right)$ states with constraint. All energies are in units of $e^{2} / \varepsilon l$. 
In summary, we have presented analytical calculations of gaps based on our new formalism, which describes the physics directly in terms of the true quasiparticles of the system, the CFs. Central to the analysis is the hamiltonian Eqn. (22) and electronic density Eqn. (11) expressed in terms of $\mathrm{CF}$ variables. The fact that many nonperturbative effects like charge and mass renormalization are built in facilitates perturbative approximations. We find agreement with with numerical results to within $25 \%$ (for the physical value of $\lambda$ [26, 30]) upon treating the constraint in the simplest manner. In higher density samples, where the effective thickness is larger, and the magnetic length smaller, $\lambda=\Lambda / l$ will be larger. As $\lambda$ increases, we expect our effective theory will become more accurate, since we are using $\bar{\rho}$ matrix elements for a smaller range of $q$. We should bear in mind that fluctuation corrections beyond HF may become important for large $\lambda$, since the higher CF-LLs come very close to the first unoccupied one. We also expect to run into problems as we approach $\nu=1 / 2$, since constraints are known to play a crucial role in this limit [34, 20,35].

Many other physical quantities in the gapped fractions can be computed using this formalism, such as the magnetoexciton dispersion [23, 19, 36 38], which we will present elsewhere. Since we have a concrete microscopic description of the CFs, we can also couple them to impurity and edge potentials.

We would like to thank S.H.Simon, S.M.Girvin, and K.Park for helpful conversations, and especially J.K.Jain for numerous insightful discussions and for releasing his results prior to publication. R.S. is grateful for support from the NSF (DMS98-00626).

[1] J.K.Jain, Phys. Rev. Lett. 63, 199, (1989); Phys. Rev. B 41, 7653 (1990); Science 266, 1199 (1994).

[2] D.Tsui, H.Stromer and A.Gossard, Phys. Rev. Lett. 48, 1599, (1982).

[3] J.K.Jain and R.K.Kamilla, to appear in "Composite Fermions", Olle Heinonen, Editor.

[4] S.M.Girvin, in Chapter 9 of The Quantum Hall Effect, Edited by R.E.Prange and S.M Girvin, Springer-Verlag, 1990.

[5] S.M.Grivin and A.H.MacDonald, Phys. Rev. Lett. 58, 1252 (1987).

[6] S.-C.Zhang, H.Hansson and S.A.Kivelson, Phys. Rev. Lett. 62, 82, (1989); D.-H.Lee and S.-C.Zhang, Phys. Rev. Lett. 66, 1220 (1991); S.-C.Zhang, Int. J. Mod. Phys., B6, 25 (1992).

[7] N.Read, Phys. Rev. Lett., 62, 86 (1989).

[8] A.Lopez and E.Fradkin, Phys. Rev. B 44, 5246 (1991), ibid 47, 7080, (1993), Phys. Rev. Lett. 69, 2126 (1992).

[9] V.Kalmeyer and S.-C.Zhang, Phys. Rev. B 46, 9889 (1992).

[10] B.I.Halperin, P.A.Lee and N.Read, Phys. Rev. B 47, 7312 (1993).
[11] R.Shankar and G.Murthy, Phys. Rev. Lett. 79, 4437, (1997).

[12] G.Murthy and R.Shankar, to appear in "Composite Fermions", Olle Heinonen, Editor (cond-mat 9802244).

[13] D.Bohm and D.Pines, Phys. Rev. 92, 609, (1953).

[14] R.B.Laughlin, Phys. Rev. Lett. 50, 1395, (1983)

[15] S.H.Simon, A.Stern, and B.I.Halperin, Phys. Rev. B 54, R11114 (1996).

[16] S.H.Simon and B.I.Halperin, Phys. Rev. B 48, 17368, (1993).

[17] N.Read, Semi. Sci. Tech. 9, 1859 (1994); Surf. Sci., 361/362, 7 (1996).

[18] W.Kohn, Phys. Rev 123, 1242 (1961).

[19] S.M.Girvin, A.H.MacDonald and P.Platzman, Phys. Rev. B 33, 2481, (1986).

[20] D.H.Lee, Phys. Rev. Lett. 80, 4745 (1998).

[21] V.Pasquier and F.D.M.Haldane, cond-mat 9712169.

[22] F.F.Fang and W.E.Howard, Phys. Rev. Lett. 16, 797 (1966); T.Ando, A.B.Fowler, and F.Stern, Rev. Mod. Phys. 54, 437 (1982).

[23] F.D.M.Haldane and E.H.Rezayi, Phys. Rev. Lett. 54, 237 (1985).

[24] F.C.Zhang and S.Das Sarma, Phys. Rev. B 33, 2903 (1986); D.Yoshioka, J. Phys. Soc. Jpn. 55, 885 (1986); T.Chakraborty, P.Pietlainen, and F.C.Zhang, Phys. Rev. Lett, 57, 130 (1986); R.Morf and B.I.Halperin, Z. Phys. B 68, 391 (1987); R.Price and S.Das Sarma, Phys. Rev. B 54, 8033 (1996).

[25] V.Melik-Alaverdian and N.E.Bonesteel, Phys. Rev. B 52, R17032 (1995); V.Melik-Alaverdian, N.E.Bonesteel, and G.Ortiz, Phys. Rev. Lett., 79, 5286 (1997).

[26] K.Park and J.K.Jain, preprint.

[27] A.L.Fetter, C.B.Hanna, and R.B.Laughlin, Phys. Rev. B 39, 9679 (1989); ibid 43, 309 (1991); Q.Dai, J.L.Levy, A.L.Fetter, C.B.Hanna, and R.B.Laughlin, Phys. Rev. B 46, 5642 (1992).

[28] I.V.Lerner and Yu.E.Lozovik, Sov. Phys. JETP 57, 588 (1980).

[29] Note that commonly used Fang-Howard potential 22 does not have all moments finite; this is why we have focused on the model potential.

[30] R.R.Du, H.L.Störmer, D.C.Tsui, L.N.Pfeiffer, and K.W.West, Phys. Rev. Lett. 70, 2944 (1993).

[31] M.W.Ortalano, S.He, and S.Das Sarma, Phys. Rev. B 55, 7702 (1997).

[32] G.Murthy, K.Park, R.Shankar, and J.K.Jain, in preparation.

[33] G.Dev and J.K.Jain, Phys. Rev. Lett. 69, 2843 (1992).

[34] B.I.Halperin and A.Stern, Phys. Rev. Lett. 80, 5457 (1998); G. Murthy and R.Shankar, Phys. Rev. Lett. 80, 5458 (1998).

[35] N.Read, cond-mat 9804294.

[36] N.d'Ambrumenil and R.Morf, Phys. Rev. B 40, 6108 (1989); P.M.Platzman and S.He, Phys. Rev. B 49, 13674 (1994); S.He, S.H.Simon, and B.I.Halperin, Phys. Rev. B 50, 1823 (1994).

[37] K.W.Chiu and J.J.Quinn, Phys. Rev. B 9, 4724 (1974); Yu.A.Bychkov, S.V.Iordanskii, and G.M.Eliashberg, JETP Lett. 33, 152 (1981); C.Kallin and B.I.Halperin Phys. Rev B 30, 5655, 1984; S.H.Simon and B.I.Halperin, Phys. Rev. B 50, 1807 (1994).

[38] X.G.Wu and J.K.Jain, Phys. Rev. B 51, 1752 (1995); R.K.Kamilla, X.G.Wu, and J.K.Jain, Phys. Rev. Lett. 
76, 1332 (1996); Phys. Rev. B 54, 4873 (1996). 